\title{
Linear correlation models for the redox potential of organic molecules in aqueous solutions
}

Jessica C. Ortiz-Rodríguez, Juan A. Santana and Dalvin D. Méndez-Hernández*

Department of Chemistry, University of Puerto Rico at Cayey, Cayey, Puerto Rico 00736, United States.

*Corresponding author: dalvin.mendez@upr.edu

ORCID: Dalvin D. Méndez-Hernández (0000-0001-5659-415X), Juan A. Santana (0000-0003-2349-

\section{ABSTRACT}

In this study we use the molecular orbital energy approximation (MOEA) and the energy difference approximation (EDA) to build linear correlation models for the redox potentials of 53 organic compounds in aqueous solutions. The molecules evaluated include nitroxides, phenols and amines. Both the MOEA and EDA methods yield similar correlation models, however the MOEA method is less computationally expensive. Correlation coefficients $\left(\mathrm{R}^{2}\right)$ below 0.3 and mean absolute errors above $0.25 \mathrm{~V}$ were found for correlation models built without solvent effects. When explicit water molecules and a continuum solvent model are added to the calculations, correlation coefficients close to 0.8 are reached and mean absolute errors below $0.18 \mathrm{~V}$ are obtained. The incorporation of solvent effects is necessary for good correlation models, particularly for redox processes of charged molecules in aqueous solutions. A comparison of the correlation models from different methodologies is provided.

\section{KEYWORDS}

Redox potentials, aqueous solutions, linear correlations, explicit solvation 


\section{INTRODUCTION}

Energy technologies such as organic solar cells [1, 2], light-emitting diodes [3], dye-sensitized solar cells [4-11], and artificial photosynthetic systems [12-23] rely on the reduction and oxidation (redox) of different organic molecules. The calculation of the redox potential of organic molecules in solutions is crucial for the rational design of such technologies. Various methodologies are currently employed to calculate redox potential in solutions: $i)$ the Born-Haber thermodynamic cycle (BHTC) [24-27], ii) the $\mathrm{S}_{0}-\mathrm{D}_{0}$ energy difference approximation (EDA) [28-30], and iii) the molecular orbital energy approximation (MOEA) $[28,31,32]$. In the BHTC method, the redox potential is calculated from the standard free energy of product and reactants in a redox half-reaction. In the EDA and MOEA methods, a different approach is used where calculated total (or frontier molecular orbital) energies are used to build a correlation model with available experimental redox potentials. The built correlation model (typically a linear model) is then used to calculate the unknown redox potential of a given molecule from calculated total (or frontier molecular orbital) energies. In the EDA method, the total energy of the initial and final ground states in the redox process is used to build the correlation models. For the MOEA method, the energies of the frontier molecular orbitals of the $\mathrm{S}_{0}$ state are employed to build the correlation models; the energy of the lowest unoccupied molecular orbital (LUMO) and highest occupied molecular orbital (HOMO) are used for the reduction and oxidation processes, respectively.

Each of these methodologies has advantages and disadvantages. The BHTC does not require experimental data, but in turn it is the most computationally expensive. On the other hand, the EDA and MOEA methodologies require much less computational effort, but they require an experimental data set to build the correlation model, making the built model specific to the experimental conditions. However, the EDA and MOEA methods have been shown to work for a diverse set of molecules in a given solvent [27-33]. For example, Gillmore et al. [29, 30] and Méndez-Hernández et al. [31, 32] have reported strong correlations for a set of 74 diverse molecules in acetonitrile using the EDA and 
MOEA methodologies, respectively. The great advantage of the MOEA method is that once the correlation model is available only a minimal computational effort is required to obtain an unknown redox potential, which becomes crucial for high-throughput screening.

In the present work, we employ the EDA and MOEA methods to build correlation models for the redox potential of a set of 53 diverse organic compounds in aqueous solution. The set of molecules includes 3 molecular groups (nitroxides and derivatives, phenols and derivatives and amines and derivatives). In aqueous solution, the formation of strong hydrogen bonds between solvent and solute molecules makes the simulation more complex and explicit solvating water molecules are often needed to increase the accuracy of electronic structure calculations $[34,35]$. To account for hydrogen bonding effects, we have included up to 2 explicit water molecules in our models. We find that the EDA and MOEA methods yield redox potential in good agreement with the BHTC method [26]. We found that the addition of at least one explicit water molecules in conjunction with a continuum solvent model is enough for good correlation models.

\section{METHODS}

Calculations were carried out using the ORCA software package [36, 37]. Electronic structure calculations were performed within the Density Functional Theory (DFT) using the B3LYP [38, 39] functional and the $6-31++\mathrm{G}^{* *}$ basis set [40-45] as this combination has been shown to be good for organic molecules [46], particularly when hydrogen bonds are present [47, 48]. A total of 53 diverse molecules were studied, see Figure 1 . The set of molecules was originally compiled by Marenich et al. [26], and we have used the same set for convenience and consistency. Furthermore, this is a suitable set because redox potential data in aqueous solution studies is much more sparse than typical benchmarking data sets $[3,28,49-53]$. The experimental potentials of the set of 53 molecules range from 0.24 to $1.47 \mathrm{~V}$ vs the standard hydrogen electrode (SHE). Each molecular geometry was initially optimized in the gas phase. After such optimization, a series of calculations were performed to test various 
aqueous solution models. To model the effects of strong hydrogen bonds, one and two explicit water molecules were included. The various molecular models are labeled as Model A, Model B, and Model $\mathrm{C}$, for calculations without explicit water molecules, and with one and two water molecules, respec-

tively. For the Model A, Model B and Model C, calculations were performed in gas phase, and with the conductor-like screening model approach COSMO [54] and the density-based solvation model SMD [55] as implicit continuum solvent models.

The results of the various calculations were employed to build linear correlation models within the EDA and MOEA methodologies. The linear models were generated from a linear regression analysis using the experimental redox potentials, taken in V vs. SHE from Ref. [26]. The slope and intercept for each linear model as well as the mean absolute error (MAE) and Maximum deviation value (Max. Dev.) are included in Table 1 for each combination of molecular and solvation models. Additionally, we include in Table 1 results from calculations performed with and without geometrical optimization within the SMD solvation model. Such data set will help to establish if geometrical optimization with the implicit solvation models is required for reliable correlation models.

\section{RESULTS AND DISCUSSION}

We first examine the linear correlation built from calculations with Model A in the gas phase and EDA method. The parameters for this model are included in Table 1 as Correlation 1. The correlation coefficient $\left(\mathrm{R}^{2}\right)$ is only 0.28 for Correlation 1, with MAE and Max. Dev. values of 0.29 and 0.86 $\mathrm{V}$, respectively. The correlation model is also poor $\left(\mathrm{R}^{2}<0.05\right)$ if the MOEA method is used instead of EDA, see Correlation 2 in Table 1 and Figure 2A. As a baseline, a correlation model [26] built with the more general and accurate BHTC method yield a $\mathrm{R}^{2}$ value of 0.86 , and MAE and Max. Dev. of 0.26 and $0.64 \mathrm{~V}$, respectively. In general, the EDA and MOEA methods yield poor correlation models when build from calculations with Model A in the gas phase. The main reason for the low $\mathrm{R}^{2}$ values for 
calculations in the gas phase is likely the fact that our experimental potentials correspond to the reduction process of neutral molecules as well as molecular cations under aqueous conditions. We found similar poor correlations $\left(\mathrm{R}^{2}<0.65\right)$ in a previous work [31] when the set of experimental potentials were for reduction processes of both neutral and charged molecules. Simulations of reduction processes of charged molecules without including solvation effects (gas phase calculations) fail to reproduce the measured potentials. On the other hand, correlation models built from calculations in the gas phase result in excellent agreement $\left(\mathrm{R}^{2}>0.97\right)[31,32]$ when the reduction or oxidation processes are for neutral molecules, i.e. all molecules in the set undergo reduction from the neutral singlet $\mathrm{S}_{0}$ state to the charged doblet $\mathrm{D}_{0}$ state.

Correlation 3 in Table 1 corresponds to the linear model built from calculations with Model A using the continuum solvent model COSMO and the EDA method. A significant improvement is found for the linear correlation model, with $\mathrm{R}^{2}=0.74$, and MAE and Max. Dev. values of 0.19 and $0.65 \mathrm{~V}$, respectively. If the SMD solvation model is employed instead of COSMO, a slightly better correlation model is obtained, see Correlation 4 in Table 1. The MOEA method with Model A and SMD yields a correlation model (Correlation 5) with slightly lower $\mathrm{R}^{2}=0.77$ and higher MAE and Max. Dev. of 0.18 and $0.53 \mathrm{~V}$, respectively. Thus, including solvation effects with a continuum model yield $\mathrm{R}^{2}, \mathrm{MAE}$ and Max. Dev. comparable with the correlation model from the BHTC method $[26]\left(\mathrm{R}^{2}=0.86, \mathrm{MAE}=\right.$ $0.26 \mathrm{~V}$ and Max. Dev. $=0.64 \mathrm{~V}$ ). The incorporation of solvent effects is necessary for good correlation models. This is consistent with previous reports $[27,31]$ and it is especially important when dealing with species with different charges as discussed above. The results discussed here show that when solvation effects are accounted for, the EDA and MOEA methods yield similar results. We will focus on the SMD model and the MOEA method in what follows as this combination seems a reasonable compromise between accuracy and computational effort. 
As mentioned previously, strong hydrogen bonds are formed in aqueous solution. We explore the effect of including explicit water molecules hydrogen bonded to the organic molecules. The linear correlation built from calculations with model with one water molecule (Model B), SMD and the MOEA method is Correlation 6 in Table 1 (see Figure 2B). The $\mathrm{R}^{2}$, MAE and Max. Dev. are 0.81, 0.16 $\mathrm{V}$ and $0.66 \mathrm{~V}$, respectively. These results are comparable to the results obtained with the BHTC method and are slightly better than the results obtained with Model A (Correlations 5). Thus, addition of one explicit water molecule results in better correlation models, while addition of a second water molecule (Model C) does not furthers improve the correlation model (see Correlation 7 in Table 1).

The main objective of our work is to find a methodology for the high-throughput accurate prediction of redox potential for many molecules in aqueous solutions. To build the correlation models discussed above, geometry optimizations in the presence of a continuum solvent model are required. An alternative less computationally expensive is the inclusion of the implicit solvent model in a molecular geometry optimized only in the gas phase. This protocol is on average 7 times less computationally expensive than optimizing the geometry in the presence of the solvent model. Correlation 8 in Table 1 shows the correlation model built with this protocol. The resulting correlation model is similar to the one from optimizing the geometry in the presence of a solvent model (Correlation 6).

Finally, Figure 3 shows the MAE and Max. Dev. for the MOEA (Correlation 6) and BHTC [26] methods for the 3 molecular groups (nitroxides and derivatives, phenols and derivatives and amines and derivatives). MAE and Max. Dev. values depend on both the method and molecular group. For instance, MOEA yields MAE and Max Dev. values that are higher for nitroxides, lower for phenols and comparable for amines to the values from BHTC. The only group for which the BHTC method gives better results than MOEA is the nitroxides, which only has 7/53 molecules. Therefore, the larger MAE for this group could result from such underrepresentation in the data set. 
The present results show that the MOEA method is a good and computationally inexpensive alternative to build linear correlation models for the redox potential of organic molecules in aqueous solutions when solvation effects are included with continuum solvation models. However, the $\mathrm{R}^{2}$ of the built linear model are below 0.85 . We have employed the MOEA method before [31, 32] to build a linear correlation model for the redox potential of organic molecules in non-aqueous solutions and, in such cases, the $\mathrm{R}^{2}$ is above 0.99 . The difference could come from the variance of the experimental reduction potentials in aqueous and non-aqueous solutions. Reduction potentials measured from pulsed radiolysis typically have an uncertainty of $0.02 \mathrm{~V}$ [56]. However, reports from voltammetry measurements rarely indicate the experimental error, making it hard to access the uncertainty of reduction potentials from such measurements. Furthermore, the one-electron reduction of amines and phenols in aqueous solutions involves changes in the protonation state [26]. The reduction potentials of such molecules are measure indirectly by combining the experimental redox potentials of the radical species and the experimental pKas of the neutral and radical species, adding uncertainty to the experimental reduction potentials [56]. The conversion of the measured potentials from a reference electrode, like the $\mathrm{Ag} / \mathrm{AgCl}$ and saturated calomel electrodes to $\mathrm{SHE}$ also contributes to the experimental uncertainty [57]. On the other hand, differences between the $\mathrm{R}^{2}$ values of correlation models for reduction potentials in aqueous and non-aqueous solutions could be coming from the methods employed to simulate solvation effects, like continuum solvent models [26]. There is some ambiguity related to inclusion of the experimental solvation free energies of ionic species and its contribution associated with the surface potential of the solvent, which has been estimated to be about $0.14 \mathrm{~V}$ for water [58, 59]. Moreover, there are typically strong hydrogen bonds in aqueous solutions, which is not properly described by continuum solvent models. Guerard and Arey[56] have calculated the redox potentials of 22 neutral organic compounds in aqueous solution for which accurate experimental data was available and found MAE ranging from 0.270 to $0.500 \mathrm{~V}$ depending on the computational model. Clearly, better 
computational methodologies are necessary for the simulation of aqueous solvents. Possible alternatives have been proposed by Merenich et al.[26], who suggested, for example, incorporation of side reactions and the reduction/oxidation of the solvent. To reduce the uncertainties coming from the experimental data, redox potentials for a larger set of molecules are necessary. The experimental reference redox couple should be measured under identical experimental conditions in aqueous solvent to reduce uncertainty from the reference electrode [24].

\section{CONCLUSIONS}

We have used the molecular orbital energy approximation and the energy difference approximation methods in combination with implicit solvent models and explicit water molecules to build linear correlation models of the redox potential of 53 organic molecules in aqueous solutions. Our results show that incorporation of solvent effects is necessary for good correlation models. Such effects can be included with implicit solvent models, but at least one explicit water molecule is required for more accurate correlation models. The data and analysis presented herein could be useful for the highthroughput prediction of redox potential for many molecules in aqueous solutions and for the training of machine learning models.

\section{ACKNOWLEDGEMENTS}

This work was supported by the 2016-2018 Start-Up funds and the "Fondo Institucional para el Desarrollo de la Investigación (FIDI)" of the University of Puerto Rico at Cayey. J.C.O.R. was supported in part by the National Institute of General Medical Sciences of the National Institutes of Health under Award Number R25GM059429-21 and the Puerto Rico Louis Stokes Alliance For Minority Participation (PR-LSAMP). Computational resources were provided by the High-Performance Computing Facility at the University of Puerto Rico, under the Puerto Rico INBRE grant P20GM103475 from the National Institute for General Medical Sciences (NIGMS), a component of the National Institutes of 
Health (NIH) and awards 1010094 and 1002410 from the Experimental Program to Stimulate Competitive Research (EPSCoR) program of the National Science Foundation (NSF). The content is solely the responsibility of the authors and does not necessarily represent the official views of the National Institutes of Health.

\section{FIGURES AND TABLES}
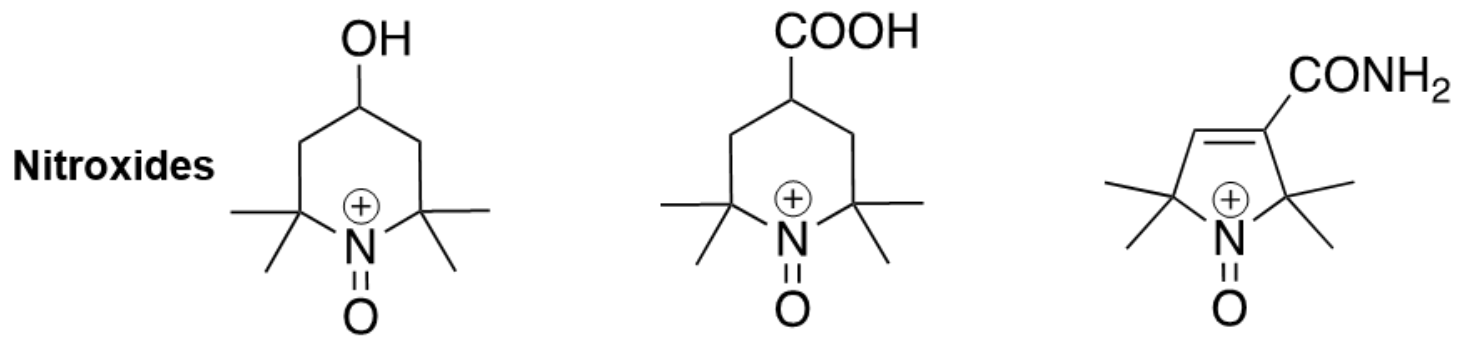

Phenols<smiles>Oc1ccccc1</smiles><smiles>COc1ccc(O)cc1</smiles><smiles>Cc1cc(O)c2ccccc2c1O</smiles>

Amines<smiles>CNC</smiles><smiles>Nc1ccccc1</smiles><smiles>Cc1c[nH]c2ccccc12</smiles>

Figure 1. Selected organic compounds from the set of 53 molecules organized by chemical groups. For a complete list of the molecules see Reference [26]. 

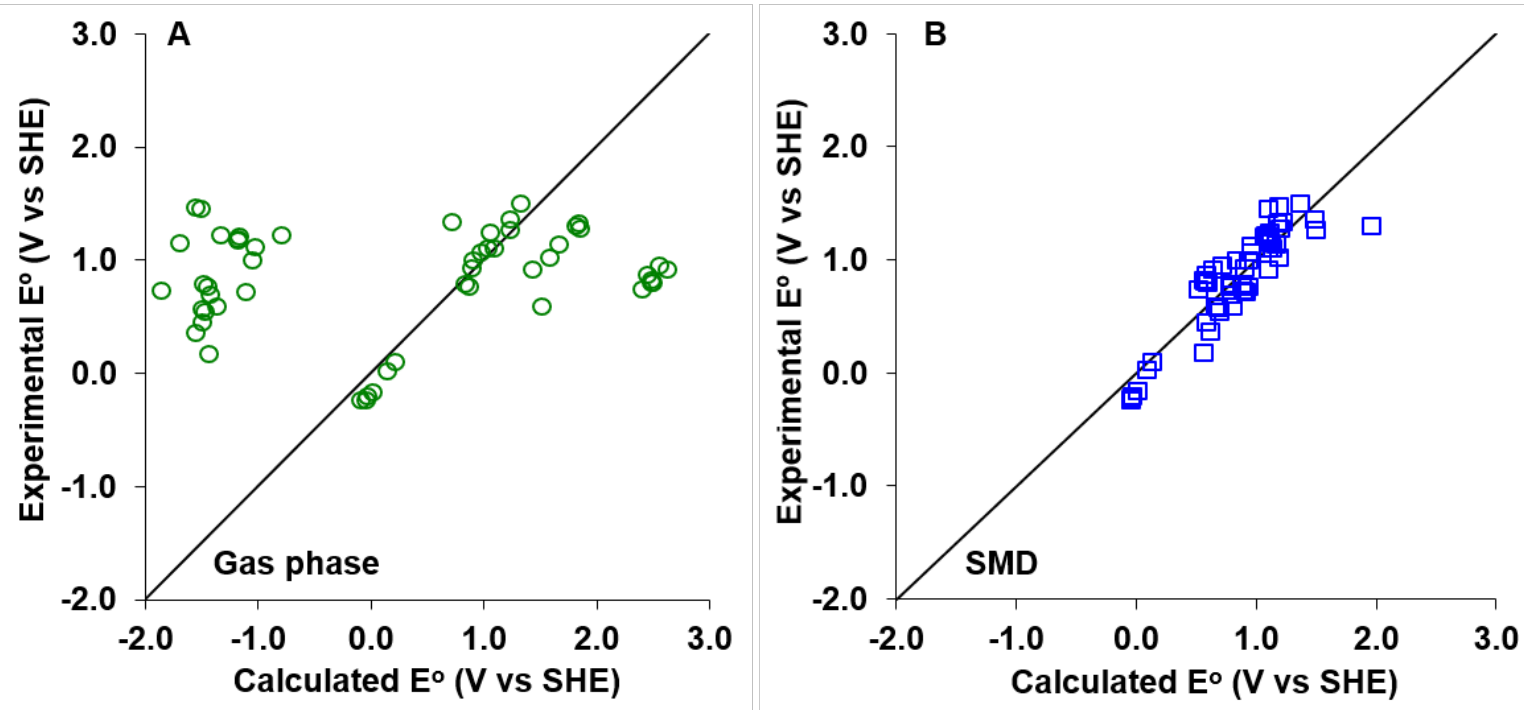

Figure 2. Comparison of correlation models built from calculations A) without and B) with SMD solvent effects. Panel A and B correspond to Correlations 2 and 6 in Table 1.
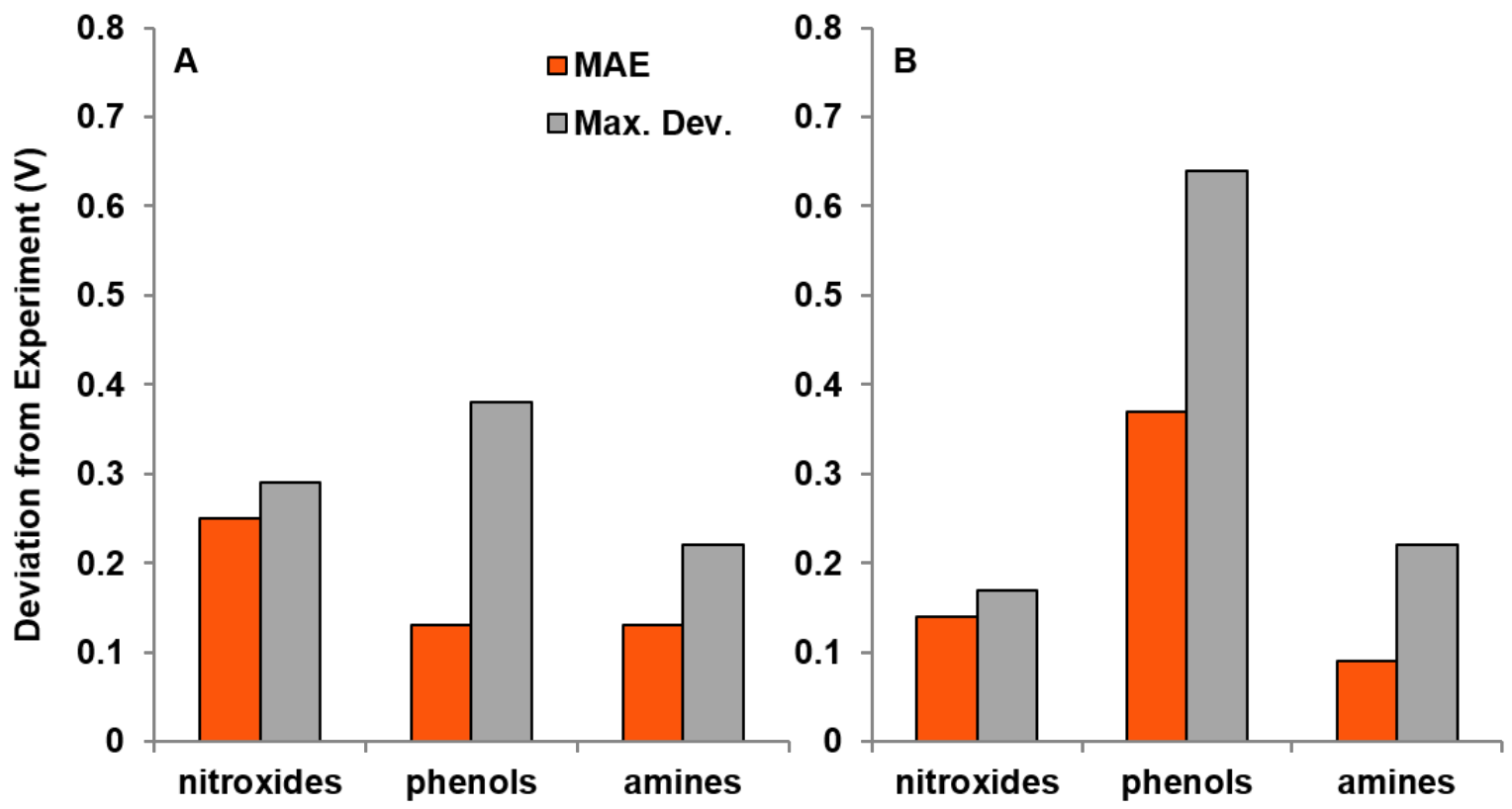

Figure 3. Mean absolute error (orange bars) and maximum deviation (gray bars) of redox potentials for each molecular group (nitroxides, phenols and amines) calculated with the (a) MOEA and (b) BHTC methodology from Reference [26] using SMD and G3(MP2,CC)(+) level of theory. Results for MOEA are from Correlation 6 in Table 1. 
Table 1. Parameters of the built linear correlation models.

\begin{tabular}{|c|c|c|c|c|c|c|c|c|}
\hline $\begin{array}{c}\text { Corre- } \\
\text { lation }\end{array}$ & Method $^{\mathbf{a}}$ & Model $^{\mathbf{b}}$ & Calculation $^{\mathbf{c}}$ & $\mathbf{R}^{\mathbf{2}}$ & $\begin{array}{c}\text { MAE } \\
(\mathbf{V})^{\mathbf{e}}\end{array}$ & $\begin{array}{c}\text { Max. } \\
\text { Dev. (V) }^{\mathbf{f}}\end{array}$ & $\begin{array}{c}\text { Slope } \\
\mathbf{( V / e V )}\end{array}$ & $\begin{array}{c}\text { Intercept } \\
(\mathbf{V})\end{array}$ \\
\hline $\mathbf{1}$ & EDA & A & Gas & 0.28 & 0.29 & 0.86 & -0.09207 & 0.4119 \\
\hline $\mathbf{2}$ & MOEA & A & Gas & 0.03 & 0.34 & 1.07 & -0.02472 & 0.7484 \\
\hline $\mathbf{3}$ & EDA & A & COSMO & 0.74 & 0.19 & 0.65 & -0.6375 & -2.245 \\
\hline $\mathbf{4}$ & EDA & A & SMD & 0.80 & 0.16 & 0.49 & -0.7587 & -2.781 \\
\hline $\mathbf{5}$ & MOEA & A & SMD & 0.77 & 0.18 & 0.53 & -0.4411 & -1.440 \\
\hline $\mathbf{6}$ & MOEA & B & SMD & 0.81 & 0.16 & 0.66 & -0.4649 & -1.577 \\
\hline $\mathbf{7}$ & MOEA & C & SMD & 0.79 & 0.17 & 0.55 & -0.4641 & -1.600 \\
\hline $\mathbf{8}$ & MOEA & B & SMD-SP & 0.78 & 0.17 & 0.55 & -0.4461 & -1.476 \\
\hline
\end{tabular}

a) Methodologies employed to build the correlation models: energy difference approximation (EDA) and the molecular orbital energy approximation (MOEA). b) Model A, B and C correspond to the molecule without, and with 1 and 2 explicit water molecules, respectively. c) Geometry optimization in the gas phase (Gas), with the continuum solvent model SMD or the continuum solvent model COSMO. d) Calculations were performed with SMD and the geometry optimized in the gas phase. e) Mean absolute error (MAE). f) Maximum deviation from experimental values.

\section{REFERENCES}

1. Padula D, Simpson JD, Troisi A (2018) Combining Electronic and Structural Features in Machine Learning Models to Predict Organic Solar Cells Properties. Mater Horiz. https://doi.org/10.1039/C8MH01135D

2. Padula D, Simpson JD, Troisi A (2019) Combining electronic and structural features in machine learning models to predict organic solar cells properties. Mater Horiz 6:343-349. https://doi.org/10.1039/C8MH01135D

3. Wang D, Huang S, Wang C, et al (2019) Computational prediction for oxidation and reduction potentials of organic molecules used in organic light-emitting diodes. Org Electron 64:216-222. https://doi.org/10.1016/j.orgel.2018.10.038

4. Swierk JR, Méndez-Hernández DD, McCool NS, et al (2015) Metal-free organic sensitizers for use in water-splitting dye-sensitized photoelectrochemical cells. Proc Natl Acad Sci 112:1681-1686. https://doi.org/10.1073/pnas.1414901112

5. Alibabaei L, Brennaman MK, Meyer TJ (2018) Light-Driven Water Splitting in the Dye-Sensitized Photoelectrosynthesis Cell. In: Tian H, Boschloo G, Hagfeldt A (eds) Molecular Devices for Solar Energy Conversion and Storage. Springer Singapore, Singapore, pp 229-257

6. Li F, Fan K, Xu B, et al (2015) Organic Dye-Sensitized Tandem Photoelectrochemical Cell for Light Driven Total Water Splitting. J Am Chem Soc 137:9153-9159. https://doi.org/10.1021/jacs.5b04856

7. McCool NS, Swierk JR, Nemes CT, et al (2016) Proton-Induced Trap States, Injection and Recombination Dynamics in Water-Splitting Dye-Sensitized Photoelectrochemical Cells. ACS Appl Mater Interfaces 8:16727-16735. https://doi.org/10.1021/acsami.6b05362

8. Youngblood WJ, Lee S-HA, Kobayashi Y, et al (2009) Photoassisted Overall Water Splitting in a Visible Light-Absorbing Dye-Sensitized Photoelectrochemical Cell. J Am Chem Soc 131:926-927. https://doi.org/10.1021/ja809108y 
9. Zhao Y, Swierk JR, Megiatto JD, et al (2012) Improving the efficiency of water splitting in dye-sensitized solar cells by using a biomimetic electron transfer mediator. Proc Natl Acad Sci 109:15612-15616. https://doi.org/10.1073/pnas.1118339109

10. Ding X, Gao Y, Zhang L, et al (2014) Visible Light-Driven Water Splitting in Photoelectrochemical Cells with Supramolecular Catalysts on Photoanodes. ACS Catal 4:2347-2350. https://doi.org/10.1021/cs500518k

11. Xu P, McCool NS, Mallouk TE (2017) Water splitting dye-sensitized solar cells. Nano Today 14:42-58. https://doi.org/10.1016/j.nantod.2017.04.009

12. Chang AM, Rudshteyn B, Warnke I, Batista VS (2018) Inverse Design of a Catalyst for Aqueous CO/CO2 Conversion Informed by the NiII-Iminothiolate Complex. Inorg Chem 57:15474-15480. https://doi.org/10.1021/acs.inorgchem.8b02799

13. Sato S, Saita K, Sekizawa K, et al (2018) Low-Energy Electrocatalytic CO2 Reduction in Water over MnComplex Catalyst Electrode Aided by a Nanocarbon Support and K+ Cations. ACS Catal 8:4452-4458. https://doi.org/10.1021/acscatal.8b01068

14. Li F, Yang H, Li W, Sun L (2018) Device Fabrication for Water Oxidation, Hydrogen Generation, and CO2 Reduction via Molecular Engineering. Joule 2:36-60. https://doi.org/10.1016/j.joule.2017.10.012

15. Bi Q-Q, Wang J-W, Lv J-X, et al (2018) Selective Photocatalytic CO2 Reduction in Water by Electrostatic Assembly of CdS Nanocrystals with a Dinuclear Cobalt Catalyst. ACS Catal 8:11815-11821. https://doi.org/10.1021/acscatal.8b03457

16. La Porte NT, Martinez JF, Chaudhuri S, et al (2018) Photoexcited radical anion super-reductants for solar fuels catalysis. Coord Chem Rev 361:98-119. https://doi.org/10.1016/j.ccr.2018.01.018

17. Matheu R, Ghaderian A, Francàs L, et al (2018) Behavior of Ru-bda Water-Oxidation Catalysts in Low Oxidation States. Chem - Eur J 24:12838-12847. https://doi.org/10.1002/chem.201801236

18. Megiatto JD, Antoniuk-Pablant A, Sherman BD, et al (2012) Mimicking the electron transfer chain in photosystem II with a molecular triad thermodynamically capable of water oxidation. Proc Natl Acad Sci 109:15578-15583. https://doi.org/10.1073/pnas.1118348109

19. McKone JR, Lewis NS, Gray HB (2014) Will Solar-Driven Water-Splitting Devices See the Light of Day? Chem Mater 26:407-414. https://doi.org/10.1021/cm4021518

20. Wang L, Fan K, Daniel Q, et al (2015) Electrochemical driven water oxidation by molecular catalysts in situ polymerized on the surface of graphite carbon electrode. Chem Commun 51:7883-7886. https://doi.org/10.1039/C5CC00242G

21. Meyer TJ, Sheridan MV, Sherman BD (2017) Mechanisms of molecular water oxidation in solution and on oxide surfaces. Chem Soc Rev 46:6148-6169. https://doi.org/10.1039/C7CS00465F

22. Busch M, Fabrizio A, Luber S, et al (2018) Exploring the Limitation of Molecular Water Oxidation Catalysts. J Phys Chem C 122:12404-12412. https://doi.org/10.1021/acs.jpcc.8b03935

23. Blakemore JD, Crabtree RH, Brudvig GW (2015) Molecular Catalysts for Water Oxidation. Chem Rev 115:12974-13005. https://doi.org/10.1021/acs.chemrev.5b00122 
24. Konezny SJ, Doherty MD, Luca OR, et al (2012) Reduction of Systematic Uncertainty in DFT Redox Potentials of Transition-Metal Complexes. J Phys Chem C 116:6349-6356. https://doi.org/10.1021/jp300485t

25. Davis AP, Fry AJ (2010) Experimental and Computed Absolute Redox Potentials of Polycyclic Aromatic Hydrocarbons are Highly Linearly Correlated Over a Wide Range of Structures and Potentials. J Phys Chem A 114:12299-12304. https://doi.org/10.1021/jp106088n

26. Marenich AV, Ho J, Coote ML, et al (2014) Computational electrochemistry: prediction of liquid-phase reduction potentials. Phys Chem Chem Phys 16:15068-15106. https://doi.org/10.1039/C4CP01572J

27. Kulinich AV, Derevyanko NA, Ishchenko AA, et al (2019) Structure and redox properties of polymethine dyes: Electrochemical and DFT/TD-DFT study. Dyes Pigments 161:24-33. https://doi.org/10.1016/j.dyepig.2018.09.031

28. Gieseking RLM, Ratner MA, Schatz GC (2018) Benchmarking Semiempirical Methods To Compute Electrochemical Formal Potentials. J Phys Chem A 122:6809-6818. https://doi.org/10.1021/acs.jpca.8b05143

29. Lynch EJ, Speelman AL, Curry BA, et al (2012) Expanding and Testing a Computational Method for Predicting the Ground State Reduction Potentials of Organic Molecules on the Basis of Empirical Correlation to Experiment. J Org Chem 77:6423-6430. https://doi.org/10.1021/jo300853k

30. Speelman AL, Gillmore JG (2008) Efficient Computational Methods for Accurately Predicting Reduction Potentials of Organic Molecules. J Phys Chem A 112:5684-5690. https://doi.org/10.1021/jp800782e

31. Méndez-Hernández DD, Gillmore JG, Montano LA, et al (2015) Building and testing correlations for the estimation of one-electron reduction potentials of a diverse set of organic molecules. J Phys Org Chem 28:320-328. https://doi.org/10.1002/poc.3413

32. Méndez-Hernández DD, Tarakeshwar P, Gust D, et al (2013) Simple and accurate correlation of experimental redox potentials and DFT-calculated HOMO/LUMO energies of polycyclic aromatic hydrocarbons. J Mol Model 19:2845-2848. https://doi.org/10.1007/s00894-012-1694-7

33. Winget P, Weber EJ, Cramer CJ, Truhlar DG (2000) Computational electrochemistry: aqueous one-electron oxidation potentials for substituted anilines. Phys Chem Chem Phys 2:1231-1239. https://doi.org/10.1039/A909076B

34. Jr JDM, Méndez-Hernández DD, Tejeda-Ferrari ME, et al (2014) A bioinspired redox relay that mimics radical interactions of the Tyr-His pairs of photosystem II. Nat Chem 6:423-428. https://doi.org/10.1038/nchem.1862

35. Close DM, Wardman P (2018) Calculation of Standard Reduction Potentials of Amino Acid Radicals and the Effects of Water and Incorporation into Peptides. J Phys Chem A 122:439-445. https://doi.org/10.1021/acs.jpca.7b10766

36. Neese F (2012) The ORCA program system. Wiley Interdiscip Rev Comput Mol Sci 2:73-78

37. Neese F, Wennmohs F, Becker U, et al (2014) ORCA, version 3.0. Inst Phys Theor Chem Bonn

38. Becke AD (1993) AD Becke, J. Chem. Phys. 98, 5648 (1993). J Chem Phys 98:5648

39. Lee C, Yang W, Parr RG (1988) Phys. Re V. 1988. B37 785:789 
40. Hariharan PC, Pople JA (1972) The effect of d-functions on molecular orbital energies for hydrocarbons. Chem Phys Lett 16:217-219. https://doi.org/10.1016/0009-2614(72)80259-8

41. Hariharan PC, Pople JA (1973) The influence of polarization functions on molecular orbital hydrogenation energies. Theor Chim Acta 28:213-222. https://doi.org/10.1007/BF00533485

42. Pietro WJ, Francl MM, Hehre WJ, et al (1982) Self-consistent molecular orbital methods. 24. Supplemented small split-valence basis sets for second-row elements. J Am Chem Soc 104:5039-5048. https://doi.org/10.1021/ja00383a007

43. Krishnan RB (1980) JS; Seeger, R.; Pople. J Chem Phys 72:650

44. Krishnan R, Binkley JS, Seeger R, Pople JA (1980) Self-consistent molecular orbital methods. XX. A basis set for correlated wave functions. J Chem Phys 72:650-654

45. McLean AD, Chandler GS (1980) Contracted Gaussian basis sets for molecular calculations. I. Second row atoms, $Z=11-18$. J Chem Phys 72:5639-5648

46. Tirado-Rives J, Jorgensen WL (2008) Performance of B3LYP Density Functional Methods for a Large Set of Organic Molecules. J Chem Theory Comput 4:297-306. https://doi.org/10.1021/ct700248k

47. Pal S, Kundu TK (2012) Theoretical Study of Hydrogen Bond Formation in Trimethylene Glycol-Water Complex. In: Int. Sch. Res. Not. https:/www.hindawi.com/journals/isrn/2012/570394/. Accessed 7 Jun 2019

48. Crespo-Hernández CE, Close DM, Gorb L, Leszczynski J (2007) Determination of Redox Potentials for the Watson-Crick Base Pairs, DNA Nucleosides, and Relevant Nucleoside Analogues. J Phys Chem B 111:5386-5395. https://doi.org/10.1021/jp0684224

49. Goerigk L, Hansen A, Bauer C, et al (2017) A look at the density functional theory zoo with the advanced GMTKN55 database for general main group thermochemistry, kinetics and noncovalent interactions. Phys Chem Chem Phys 19:32184-32215. https://doi.org/10.1039/C7CP04913G

50. Hicks LD, Fry AJ, Kurzweil VC (2004) Ab initio computation of electron affinities of substituted benzalacetophenones (chalcones): a new approach to substituent effects in organic electrochemistry. Electrochimica Acta 50:1039-1047. https://doi.org/10.1016/j.electacta.2004.08.003

51. Ho J (2014) Are thermodynamic cycles necessary for continuum solvent calculation of pKas and reduction potentials? Phys Chem Chem Phys 17:2859-2868. https://doi.org/10.1039/C4CP04538F

52. Isegawa M, Neese F, Pantazis DA (2016) Ionization Energies and Aqueous Redox Potentials of Organic Molecules: Comparison of DFT, Correlated ab Initio Theory and Pair Natural Orbital Approaches. J Chem Theory Comput 12:2272-2284. https://doi.org/10.1021/acs.jctc.6b00252

53. Assary RS, Brushett FR, Curtiss LA (2014) Reduction potential predictions of some aromatic nitrogencontaining molecules. RSC Adv 4:57442-57451. https://doi.org/10.1039/C4RA08563A

54. Klamt A, Schüürmann G (1993) COSMO: a new approach to dielectric screening in solvents with explicit expressions for the screening energy and its gradient. J Chem Soc Perkin Trans 2 799-805. https://doi.org/10.1039/P29930000799 
55. Universal Solvation Model Based on Solute Electron Density and on a Continuum Model of the Solvent Defined by the Bulk Dielectric Constant and Atomic Surface Tensions | The Journal of Physical Chemistry B. https://pubs.acs.org/doi/abs/10.1021/jp810292n. Accessed 29 May 2019

56. Guerard JJ, Arey JS (2013) Critical Evaluation of Implicit Solvent Models for Predicting Aqueous Oxidation Potentials of Neutral Organic Compounds. J Chem Theory Comput 9:5046-5058. https://doi.org/10.1021/ct4004433

57. Aqueous and Surface Redox Potentials from Self-Consistently Determined Gibbs Energies | The Journal of Physical Chemistry C. https://pubs.acs.org/doi/abs/10.1021/jp802627s. Accessed 4 Jun 2019

58. Fawcett WR (2008) The Ionic Work Function and its Role in Estimating Absolute Electrode Potentials. Langmuir 24:9868-9875. https://doi.org/10.1021/1a7038976

59. Hunenberger P, Reif M (2011) Single-Ion Solvation

\section{GRAPHICAL ABSTRACT}
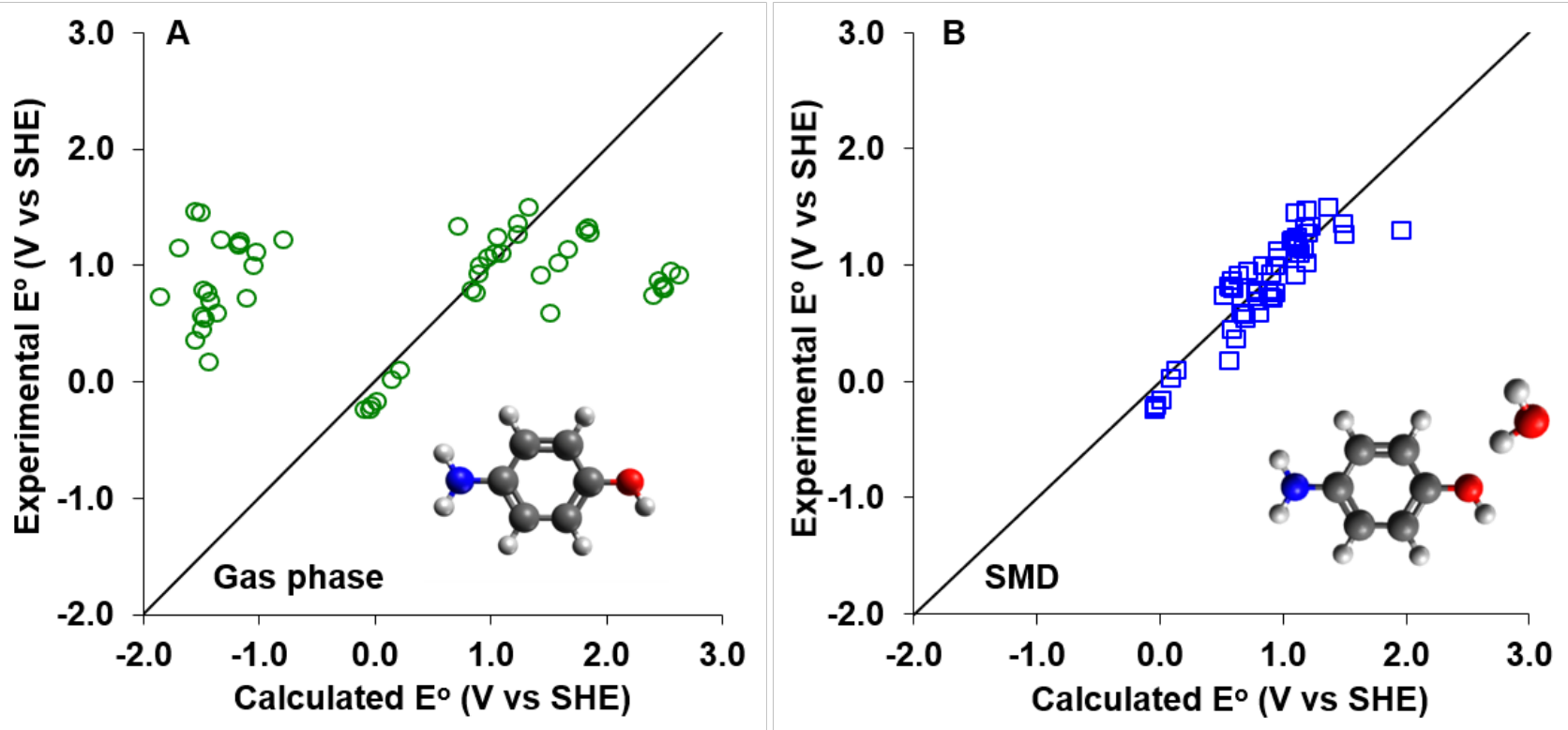